\title{
Policy Challenges and Opportunities to Address Changing Paradigms in Cancer Care Delivery
}

\author{
Katy Winckworth-Prejsnar, MPHa; James McCanney, JDa; Alyssa A. Schatz, MSWa; Warren Smedley, MSHA, MSHOSb; \\ Leonidas C. Platanias, MD, PhDc; Cecil M. Benitez, PhDd; Lee N. Newcomer, MD, MHA;
}

C. Lyn Fitzgerald, MJa; and Robert W. Carlson, MDa,f

\begin{abstract}
Multiple factors are forcing the healthcare delivery system to change. A movement toward value-based payment models is shifting these systems to team-based integration and coordination of care for better efficiencies and outcomes. Workforce shortages are stressing access and quality of care for patients with cancer and survivors, and their families and caregivers. Innovative therapies are expensive, forcing payers and employers to prioritize resources. Patients are advocating for care models centered on their needs rather than those of providers. In response, payment policies have recently focused on the promotion of alternative payment models that incentivize coordinated, high-quality care with consideration for value and controlling the increasing overall costs associated with cancer and its treatment. Given the multitude of factors confounding cancer care, NCCN convened a multistakeholder working group to examine the challenges and opportunities presented by changing paradigms in cancer care delivery. The group identified key challenges and developed policy recommendations to address 4 high-visibility topics in cancer care delivery. The findings and recommendations were then presented at the NCCN Policy Summit: Policy Challenges and Opportunities to Address Changing Paradigms in Cancer Care Delivery in September 2018, and multistakeholder roundtable panel discussions explored these findings and recommendations along with additional items. This article encapsulates the discussion from the NCCN Working Group meetings and the NCCN Policy Summit, including multistakeholder policy recommendations on delivery issues in cancer care designed to help inform national policies moving forward.
\end{abstract}

J Natl Compr Canc Netw 2019;17(5):424-431 doi: $10.6004 /$ jnccn.2019.7299

\footnotetext{
aNational Comprehensive Cancer Network, Plymouth Meeting, Pennsylvania; bUAB Health System, Birmingham, Alabama; ${ }^{\text {}}$ Robert H. Lurie Comprehensive

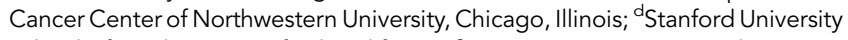
School of Medicine, Stanford, California; ' $L$ ee N. Newcomer Consulting, Minnetonka, Minnesota; and ${ }^{\mathrm{f}}$ Fox Chase Cancer Center, Philadelphia, Pennsylvania.
}

\section{Executive Summary}

The US healthcare delivery system is highly complex, confounded by a multitude of factors, from rapid breakthroughs in clinical treatments to the substantial redesign of workflows into value-based care models, and shifting economic incentives. National policies, however, do not always keep pace with these changes or accurately reflect the daily challenges of cancer care delivery and best practices. Simultaneously, workforce shortages could significantly affect access and quality of care for patients with cancer and survivors, as well as their families and caregivers. Given the rapidly increasing costs of care delivery, the pace of innovative yet expensive therapies and interventions, and the importance of patient-centered care, policy solutions have recently centered on the promotion of alternative payment models that incentivize coordinated, high-quality care with consideration for value and controlling the increasing overall costs of cancer care delivery. Although significant enthusiasm exists regarding value-based payment and delivery, value-based care remains difficult to define, bring to scale, and measure for success across varied stakeholder groups. It is important to analyze the cost trend elements of successful value-based care to sustain the overall healthcare system, and it will require additional policy recommendations to address the high costs of cancer care.

NCCN convened a multistakeholder working group (see supplemental eAppendix 1, available with this article at JNCCN.org) to examine the challenges and opportunities presented by these changing paradigms in cancer care delivery. The group identified key challenges and developed policy recommendations to address 4 high-visibility topics in cancer care delivery: (1) valuebased payment models, (2) team-based coordination of cancer care and scope of practice, (3) innovative treatments, and (4) shortages and diversity in the workforce.

See JNCCN.org for supplemental online content. 
These findings and recommendations from the working group were then presented in September 2018 at the NCCN Policy Summit: Policy Challenges and Opportunities to Address Changing Paradigms in Cancer Care Delivery. Expert speakers and multistakeholder panel discussions explored these findings and recommendations along with additional items. This article summarizes the collective expert policy recommendations (Table 1) on delivery issues in cancer care, designed to help inform national policies moving forward.

\section{Delivering Care Under Value-Based Payment Models}

Defining value in a way that is meaningful and useful to a variety of diverse stakeholders is a significant challenge. "Value" is often understood as benefits of treatment or quality of care weighed against the economic cost of care. Varying definitions of value exist among patients, providers, payers, industry, employers, and other stakeholders, with differing assumptions about value in terms of treatment experience and measurement of costs within value frameworks. The NCCN Working Group recommended the development of standardized metrics and a shared definition of value in collaboration with providers and patients. ${ }^{1}$ Moreover, given the multidisciplinary perception of value, the value discussion might require a library of concepts rather than one specific definition. ${ }^{2}$

Among private and public payers, value-based payment models have produced mixed results for cost savings, changes to quality, and coordination of patient care. During his tenure at UnitedHealthcare, Dr. Lee Newcomer, a keynote speaker at the NCCN Policy Summit, oversaw the implementation of 2 value-based care initiatives: bundled payments and gainsharing arrangements referred to as "episode payments." ${ }^{3}$ Although satisfaction was generally high with bundled arrangements between UnitedHealthcare and large academic cancer centers, only a small number of patients fit into the tightly defined bundle categories. As a result, the model lacked enough patients to assess the economic impact of the demonstration adequately. However, expanding the definition of the bundle increased the cost variation and therefore the risk for the provider. Dr. Newcomer noted that scalability can be a significant challenge for bundled payment models in the future because of the trade-off between risk and population size. ${ }^{3}$

Under a gainsharing arrangement model, cost reductions are shared between providers and a commercial payer. UnitedHealthcare created 19 distinct episodes of care definitions for breast, colon, and lung cancers and recruited 5 large community medical practices to compare their costs for these patients with similar patients in fee-for-service (FFS) payment programs. These models experienced more success with patient accrual, and over 3 years resulted in a $34 \%$ reduction in the total cost of care, primarily driven by reduced hospitalization, a quality of care improvement. However, when UnitedHealthcare attempted to replicate this model with a new set of patients in the same medical groups, there were no reductions in costs compared with the FFS controls, thus highlighting the variations that occur even within single practices. Simultaneously, the Oncology Care Model (OCM), under the Center for Medicare \& Medicaid Innovation (CMMI), began a 5-year model to test innovative payment strategies surrounding episodes of care with the goal of reducing costs and improving quality of cancer care. Since the OCM launched in 2016, participating practices have experienced various advantages and disadvantages from implementation of the new model. For example, although OCM practices have made progress on the coordination and delivery of high-quality care, significant challenges surrounding infrastructure and the payment methodology remain a hurdle for many. ${ }^{2}$ These specific examples highlight a value-based payment landscape that experienced widely variable outcomes. Although still evolving, there are valuable lessons and policy implications for value-based care moving forward.

Several policy recommendations proposed by the NCCN Working Group, and further discussed during the policy summit, centered on the availability and exchange of meaningful data for providers and patients across the healthcare continuum, through rulemaking focused on interoperability. ${ }^{1}$ As payment models increase their emphasis on value over volume, meaningful data become critical to measure success and improvement among providers. Although stakeholders applaud the OCM for facilitating the implementation of care processes that can be measured and adapted, the pace at which the Centers for Medicare \& Medicaid Services (CMS) provides participating practices with their data makes it challenging to adapt delivery systems in a timely manner. Moreover, to truly engage in shared decision-making, patients need both the necessary tools and methods to access data about their care and the opportunity to discuss their data with the clinical team for treatment planning and coordination. Data on both patient experience and real-world applications have the potential to resolve some of the challenges of feedback mechanisms when delivering and receiving value-based oncology care. The evolution of real-world data allows for retrospective analysis and prospective evaluation, including the utilization of claims data, cancer registries, and electronic health records. As public and private entities accumulate large databases relating to patient health status and/or the delivery of healthcare routinely 


\section{Table 1. Summary of Recommendations for Stakeholders}

\section{Care Delivery Topic}

Delivering care under value-based payment models

Team-based coordination of cancer care and scope of practice

\section{NCCN Working Group Recommendations ${ }^{\mathrm{a}}$}

Develop standardized metrics and a shared definition of value in collaboration with providers and patients (professional organizations, patient advocacy groups)

Support greater EHR interoperability through rulemaking (CMS, ONC)

Provide greater transparency through the availability and exchange of claims data from CMS and private payers to providers, while also focusing on greater clarity through guidance documents regarding the regulatory hurdles (HIPAA) that might prevent the sharing of meaningful claims information (HHS, CMS, private payers)

Protect patient access to high-cost innovation and the most appropriate care within value-based frameworks via a mechanism to reimburse high-cost innovative therapies within value-based payment models (CMS)

Expand beyond the OCM to implement payment models that represent best practices in the field to promote the delivery of high-quality and high-value cancer care (eg, CMS, CMMI, PTAC)

- Encourage more collaboration between PTAC and HHS (PTAC, HHS)

- Advocate for more technical assistance from PTAC before and after proposal submissions (professional organizations, PTAC) Establish reimbursement and coding mechanisms to support team-based models of cancer care tied to improvements in outcomes (CMS)

Delivery of innovative treatment

Develop policies or guidance documents to address the administrative burdens, costs, advantages, and limitations of laboratories submitting methods to the FDA for clearance/approval of laboratory methods, and recommend circumstances when such submission may be warranted (FDA)

Develop policy publication on current landscape of LDT regulation, coverage policy, method interoperability, and clinical utility standards (CMS, FDA, professional organizations)

Outline policies to protect patient access to new, high-impact therapies that include:

- Professional organizations (eg, NCCN) tasked with developing tools, standards, and evidence-based guidance for the delivery of innovative therapies that include standards around scalability of efforts, if safe and appropriate (professional organizations)

- Establish a framework or guidance document for evaluating the clinical use of emerging precision medicine biomarkers and clinical indications (CMS, private payers, professional organizations)

- Develop dissemination mechanisms (eg, resources, education, peer-to-peer networks) for the delivery of novel therapies, as well as strategies to measure the quality of these therapies, in the community (professional organizations, academic medical centers, community practices)

- Promote importance of local availability of advanced treatments and diagnostics for training of medical staff, residents, fellows, and other healthcare professionals (HHS, HRSA, professional organizations)

- Modernize reimbursement systems, particularly in CMS, to accommodate novel therapies and advanced diagnostic tools (CMS, private payers)

- Value-based payment mechanisms to reimburse costs outside bundled payments (CMS, private payers, providers)

Shortages and diversity in the cancer care workforce

Institute and expand on loan forgiveness programs for practitioners mentoring and working in underserved communities, using other professions (eg, education, primary care physicians) as possible models (US Department of Education, HRSA)

Collaborate with professional organizations (eg, ASCO) in supporting pipeline programs and legislation that promote diversity in healthcare (US Department of Education, professional organizations, HRSA)

Interstate partnership for licensing recognition for practitioners (build from the IMLC model) (licensing bodies, state regulation) Address disparate workforce shortages through greater telemedicine polices and regulation (state societies, CMS, state regulation, professional organizations)

Institute policies to reduce and/or shift administrative documentation and reporting requirements (CMS)

Develop team-based payment models to support care team providers including, but not limited to, APPs, community health workers, patient navigators, genetic counselors, dieticians, nursing education, and pharmacists (HHS, PTAC)

- Collaborate with state societies to draft model legislation; establish best practices for expansion and buy in of state regulation (eg, oral parity laws)

Abbreviations: APPs, advanced practice providers; CMMI, Center for Medicare Medicaid Innovation; CMS, Centers for Medicare \& Medicaid Services; EHR, electronic health record; HHS, US Department of Health \& Human Services; HRSA, Health Resources and Services Administration; IMLC, Interstate Medical Licensure Compact; LDT, laboratory-developed tests; OCM, Oncology Care Model; ONC, Office of the National Coordinator for Health Information Technology; PTAC, Physician-Focused Payment Model Technical Advisory Committee.

aStakeholder groups that should have the primary responsibility for implementing each recommendation are indicated in parentheses, where applicable.

collected from a variety of sources, there are opportunities to aggregate these data to provide a better picture of how therapies perform outside of clinical trials. ${ }^{3}$ Policies are needed to provide greater transparency about the availability and exchange of claims data from CMS and private payers to providers, while also focusing on greater clarity via guidance documents regarding regulatory hurdles (ie, HIPAA) that might prevent the sharing of meaningful claims information. ${ }^{1}$
Panelists and working group members emphasized the need to expand beyond the OCM to implement payment models that represent best practices in the field in order to promote the delivery of highquality and high-value cancer care. ${ }^{1}$ The Medicare Access and CHIP Reauthorization Act of 2015 (MACRA) created incentives for physicians to participate in alternative payment models, including the development of physician-focused payment models (PFPMs) facilitated 
through the Physician-Focused Payment Model Technical Advisory Committee (PTAC). To date, most alternative payment models are focused on primary care or hospital-based procedures, with an identified need for more programs across specialties, especially cancer care, where national spending continues to increase. ${ }^{4}$ As of September 2018, PTAC has recommended 14 PFPMs to the US Department of Health \& Human Services (HHS), including 8 for limited-scale testing. Thus far, none of the models has gained approval for implementation, leading to stakeholder confusion regarding the likelihood that HHS will adopt any of these models in the future. ${ }^{2}$

The NCCN Working Group specifically outlined the need to encourage more collaboration between PTAC and HHS, and for HHS to provide more technical assistance to model developers, including appropriate payment and quality measures, to facilitate the success of PTAC-approved proposals moving forward. ${ }^{1}$ Additionally, new alternative payment model approaches will need the flexibility of testing and refinement to help identify successful models of value-based care. To provide greater clarity, and in-line with the recommendations previously discussed, HHS recently released a Fact Sheet ${ }^{5}$ with the aim of helping stakeholders better understand how HHS is using PTAC's recommendations to date, as well as additional considerations that HHS will use to prioritize PFPM development moving forward.

It will be important that policies allow for the flexibility of testing new value-based payment aproaches and are held accountable for outcomes that focus on tracking and measuring the impact of these models relative to access, resource utilization, safety, and, ultimately, outcomes of patients with cancer. Beyond value-based payment development among payers, panelists also recommended policy improvements to the fee schedule, addressing the cost of care with shifts in sites of care from private community practices to hospital-owned facilities and practices, and policies that encourage data sharing in a transparent way with both patients and practitioners. The goal is to promote patient-centric care and shared decision-making treatment plans as core elements of healthcare delivery.

\section{Team-Based Coordination of Cancer Care and Scope of Practice}

Oncology has historically had a unique focus on multidisciplinary care. Patients with cancer are frequently required to see many health specialists, including medical, surgical, and radiation oncologists; primary care physicians; genetic counselors; cardio-oncologists; and rehabilitation counselors. Compounding this, the delivery and administration of cancer care has become increasingly complex with the introduction of precision medicine approaches and other new therapies with unique sideeffect profiles, new regulatory requirements, and an increasing emphasis on psychosocial support as part of the care continuum. As the complexity of cancer care increases, and payers move toward value-based reimbursement methodologies, team-based care models become even more critical for the optimal delivery of high-quality cancer care. ${ }^{6}$

In 2013, the Institute of Medicine convened a workshop to improve the quality of cancer care, comprising oncology providers, surgeons, primary care providers, researchers, policy-makers, and patients. ${ }^{7}$ A key finding from the workshop was that high-quality cancer care delivery systems must include an adequately staffed, welltrained, and coordinated workforce. The report specifically stated that, "Members of the cancer care team should coordinate with each other and with primary/ geriatrics and specialist care teams to implement patients' care plans and deliver comprehensive, efficient, and patient-centered care." This goal was accompanied by policy recommendations that included eliminating the scope of practice and reimbursement barriers to team-based coordination of care; however, many policy and practice barriers remain that prohibit achieving truly coordinated team-based cancer care, which puts patients with cancer at risk for poorer health outcomes.

A significant barrier to the provision of team-based care coordination services is a lack of appropriate reimbursement for value-added team activities. Although there is significant momentum for value-based payment models, most healthcare providers $(86 \%-95 \%)$ are paid under a FFS payment model. ${ }^{8}$ These payments often lack support and coverage mechanisms for coordination of care across a patient's multiple providers and treatments, leading to inefficient and fragmented care that is not personalized to individual patient needs. Currently, there is a lack of adequate reimbursement for many of the important ancillary services provided by oncology support staff. A well-trained clinical team of advanced practice providers, nurses, patient navigators, community health workers, genetic counselors, pharmacists, and other nonphysician staff, among others, is vitally important. Having a strong, unified clinical team that can adequately communicate with a patient regarding the risks and benefits of different available treatment options engages the patient as an active participant in treatment design and planning. Promoting shared decision-making achieves better outcomes and results in higher patient satisfaction. ${ }^{9}$ Developing teambased payment models that appropriately reimburse practices will incentivize the hiring of a more complete, well-trained, effective team. 
In-line with the working group recommendation to establish reimbursement and coding mechanisms to support team-based models of cancer care that are tied to improvements in outcomes, ${ }^{1}$ Congressman Mark DeSaulnier of California recently introduced 2 relevant federal bills. The first, the Cancer Care Planning and Coordination Act, ${ }^{10}$ would create a reimbursement mechanism in Medicare FFS for care planning and coordination services for beneficiaries with cancer. The second, the Patient Navigation Assistance Act, ${ }^{11}$ would require Medicaid programs to reimburse for patient navigator programs. It remains unclear whether these bills will ultimately become law.

Interoperability, lack of data standardization, and reporting burdens for providers present significant challenges to achieving team-based integration and coordination of care under value-based payment models. Health information technology and electronic health record systems should be tools to facilitate efficient, coordinated care across the cancer care continuum. Various policies and initiatives, including the Health Information Technology for Economic and Clinical Health (HITECH) Act, MACRA, and others, recognize the impact that health information technology can have on cancer care. However, significant challenges remain in the ability to readily share and reconcile information to coordinate effective, efficient multidisciplinary and multisite cancer care. ${ }^{2}$ Policies that address the increasing burdens and complexity of documentation and ordering requirements help promote a greater focus on interaction with patients to make key, thoughtful healthcare decisions.

Although policy challenges remain, many academic and community cancer centers are taking the lead in creating team-based models of care to enhance and improve care coordination for patients, representing an opportunity to address areas of practice that can be standardized and scalable. At the NCCN Policy Summit, Amy Porter-Tacoronte of the Sidney Kimmel Comprehensive Cancer Center at Johns Hopkins University presented a unique patient care team model developed at her institution. In this model, patient care teams are multidisciplinary, defined as the delivery of multiple services without direct interaction, but also transdisciplinary, referring to a model that involves the use of a team approach consisting of many different types of providers who collaborate to identify and meet patient and family needs. ${ }^{12}$ The need for a transdisciplinary component became clear as Maryland transitioned to a global budget revenue (GBR) model of payment. The GBR is a new method of payment recently implemented by Maryland's all-payer system in conjunction with a Medicare waiver, which restricts the amount of revenue a hospital may generate by capping the annual budget regardless of volume. ${ }^{13}$ Under this system, the Kimmel Cancer Center increased its efforts to find additional operational efficiencies and improved coordination of care. The staff took on this challenge and developed the Johns Hopkins Medicine (JHM) Care Management Continuum, a program of care delivery and coordination that promotes quality evidence-based care; efficient use of resources, health, and maximum functional status; and seamless transitions and integrated services across the care continuum. ${ }^{7}$ The JHM Care Management Continuum aims to improve care health, care quality, and care affordability, while identifying and reducing unnecessary costs and improving overall efficiencies. ${ }^{14}$ Policy and practice challenges remain under the GBR model of reimbursement, but the Kimmel Cancer Center is taking proactive steps to ensure adequate care coordination and team-based care at all levels.

\section{Delivery of Innovative Treatments}

Precision medicine is transforming healthcare by using genetics, genomics, and big data to move beyond one-sizefits-all models of prevention, diagnosis, and treatment. As cancer care becomes increasingly more individualized and complex with precision medicine and novel treatments, including but not limited to CAR T-cell therapy, novel immunotherapies, and gene targeting approaches, there are significant policy implications for care coordination, validity of data, and coverage mechanisms.

The NCCN Working Group outlined several regulatory barriers in the laboratory-developed test (LDT) space, including variance in regulatory oversight, measurement of the clinical utility of these tests, and the high costs associated with pursuing an FDA clearance or approval. Although there is recognition that LDT regulatory reform is necessary, stakeholders disagree on policy solutions to balance access to these tests with mechanisms to ensure that they are analytically and clinically valid. A bipartisan group of House and Senate members recently introduced the Verifying Accurate, Leading-edge IVCT Development (VALID) Act that establishes a framework to oversee in vitro clinical tests (IVCTs), including LDTs. ${ }^{15}$ The draft bill aligns closely with a legislative proposal published by the FDA in late 2018 outlining processes to standardize FDA-reviewed tests and LDTs, facilitate transparency regarding the performance of LDTs, and modernize regulation of IVCTs. ${ }^{16}$ It is important that the FDA, legislators, and a broad range of stakeholders continue to develop policies or guidance documents that address the administrative burdens, costs, advantages, and limitations of laboratories submitting methods to the FDA for clearance/approval of laboratory methods and recommend circumstances in which such submissions may be warranted. ${ }^{1}$ Additionally, the 
working group recommended that CMS and the FDA collaborate with professional organizations to develop a policy publication on the current landscape of LDT regulation, coverage policy, method interoperability, and clinical utility standards. ${ }^{1}$

From the commercial payer perspective, coverage will include expensive diagnostics and therapies if there is demonstrated value. However, given the variability regarding what constitutes value and the rapid pace of evidence development around clinical use of high-cost treatments, the NCCN Working Group recommended that CMS, private payers, and professional organizations work together to establish a framework or guidance document for evaluating the clinical utility of emerging precision medicine biomarkers and clinical indications. ${ }^{1}$ In-line with this recommendation, the FDA recently announced a new group, the Office of Drug Evaluation Science, to evaluate biomarkers, bioinformatics, and patient-reported outcomes. ${ }^{17}$

To help facilitate the safe and effective administration of innovative therapies to patients, it will be important for professional societies, data entities, and private/public insurers to collaborate on tools, standards, and evidence-based guidelines for innovative therapies and testing that include standards around scalability of efforts, if safe and appropriate. ${ }^{1}$ Given the rapid pace of innovation, new treatment modalities and toxicities might not be part of standard medical education and professional training, emphasizing the importance of developing dissemination mechanisms (eg, resources, education, peer-to-peer networks) for the delivery of novel therapies, as well as strategies to measure the quality of these therapies in the clinical setting. ${ }^{1}$

Beyond safe and effective administration of new technologies, reimbursement and cost of innovative treatments are major hurdles for ensuring patient access. The high cost and outdated reimbursement mechanisms for CAR T-cell therapy, particularly among Medicare beneficiaries, has been a barrier to care for both providers and patients. Medicaid coverage varies widely by state, and reimbursement has been particularly challenging for Medicare providers in the inpatient setting, where most patients are currently treated due to the significant and unpredictable toxicities associated with CAR T-cell therapy administration. In the commercial market to date, coverage has generally been available via one-time agreements for individual patients. Recent policies from CMS, including the FY 2019 Hospital Inpatient Prospective Payment System (IPPS) proposed rule and the national coverage decision (NCD) on CAR $\mathrm{T}$-cell therapy for Medicare beneficiaries with advanced cancer, have not yet provided meaningful solutions for reimbursement challenges. Providers continue to be burdened with significant unreimbursed costs for each patient receiving CAR T-cell therapy, which is not sustainable. If not addressed, this may lead to significant barriers for many patients to access innovative therapies. In addition to CAR T-cell therapy, a significant number of cell and gene therapy products are in development, and by 2025, the FDA predicts it will be approving 10 to 20 cell and gene therapy products each year. ${ }^{18}$ Because of the rapid influx of new technologies in the pipeline, there is a need for payers to modernize reimbursement systems, particularly CMS, to accommodate novel therapies and advanced diagnostic tools. ${ }^{1}$

\section{Shortages and Diversity in the Cancer Care Workforce}

In cancer care, patients rely on specialized treatment and require support from many different clinical specialties throughout the continuum of care, from screening to diagnosis through to survivorship. The NCCN Working Group and Policy Summit speakers explored policy recommendations that address shortages and promote diversity from underrepresented communities in the cancer care workforce, building on the Association of Community Cancer Centers' strategy buckets to address workforce shortages: recruitment, retention, and integration. ${ }^{16}$ Overall, access to high-quality cancer care depends on policies that can sustain a diverse and robust workforce to support optimal cancer care for patients.

Research has shown that diversity in race/ethnicity, sex, sexual orientation, and socioeconomic status among health professions is associated with enhanced patient satisfaction, improved access for minority patients, and better provider-patient communication. ${ }^{19}$ Given these health benefits, it is important to facilitate policies that increase the number of oncologists from underrepresented racial and ethnic groups, geographic locations, and other diverse backgrounds. In oncology, just $2 \%$ of the physician workforce self-identifies as black/African American and 3\% as Hispanic/Latino. In addition, the proportion of black/African American and Hispanic/Latino oncology fellows is consistently lower than other fellowships in internal medicine subspecialties. ${ }^{20}$ In contrast to findings from previous workforce shortage studies, the overall oncology workforce has remained relatively stable; however, there is an inadequate supply of physicians in predominately rural geographic areas that could affect the ability to meet the demand of cancer services nationally. In particular, rural practices have a more difficult time recruiting physicians and support staff than their urban counterparts. For example, ASCO's The State of Cancer Care in America: 2014, found that $>70 \%$ $(n=2,067)$ of US counties analyzed had no medical 
oncologists at all. ${ }^{21}$ Using other professions (eg, education, primary care physicians) as possible models, the NCCN Working Group recommended instituting and expanding on loan forgiveness programs for practitioners mentoring and working in underserved communities and collaborating with professional organizations (eg, ASCO) to support pipeline programs that promote diversity in healthcare. ${ }^{1}$

Patients in rural areas face significant transportation issues, ranging from physical distance to the closest cancer center, to actual means of transport, or the ability to cover associated costs. ${ }^{2}$ Telehealth services have the capacity to bring healthcare into areas with limited access to high-quality care. CMS currently covers telehealth services for Medicare beneficiaries; however, statutory laws limit payment for telehealth services to a small percentage of the total Medicare patient population based on several factors, including but not limited to specified services, providers, technology, and patient locations. Another regulatory hurdle includes disparate state laws governing telehealth site definitions, covered providers, and payment parity, therefore making it challenging for the expansion of telehealth delivery across state lines.

A potential policy solution includes the interstate partnership for licensing recognition for practitioners. ${ }^{1}$ The Interstate Medical Licensure Compact (IMLC) model has developed innovative ways to care for patients across state lines. Successes of the IMLC address the challenge facing rural practices in 3 ways. First, the IMLC has made it easier for practitioners to use telemedicine for patients who cannot make every necessary appointment in person. Second, many rural practices are the only cancer clinic for hundreds of miles, meaning they serve a number of out-of-state patients. IMLC uses guidelines that every practice must adhere to, ensuring that each patient receives standardized care. Finally, IMLC teaches practices how to allow support staff to manage workloads that were previously handled by the physician and, as a result, were more costly. ${ }^{22}$ Continued interstate partnerships that build on the IMLC model are necessary to reduce shortages and diversity in the cancer care workforce. Specifically, the NCCN Working Group recommended that professional organizations like NCCN collaborate with state societies to draft model legislation and establish best practices for telemedicine expansion. ${ }^{1}$ Despite the improvements models such as the IMLC can help achieve, there remain many statutory barriers for FFS models, as well as individual state regulations, that must be addressed to maximize the benefits that telemedicine can bring.

Lastly, the administrative burden associated with fulfilling CMS reporting requirements is onerous, which contributes to physician burnout, poor retention, and system-level inefficiencies. Manual extraction is required for reporting $55 \%$ of practice data. ${ }^{1}$ Under the Merit-based Incentive Payment System (MIPS), CMS estimated that reporting requirements cost $\$ 1.3$ billion in additional staff work in the first year alone. ${ }^{23}$ Stakeholders in oncology need to collaborate with CMS to identify ways to off-load burdensome administrative requirements from the practices, while simultaneously starting to reimburse those same practices for essential services performed by support staff. $^{1}$

\section{Conclusions}

Thought leaders from the NCCN Working Group and Policy Summit collaborated on key policy areas that address challenges of delivering value-based oncology care, ensuring team-based coordination of care, emphasizing policies to ensure the adoption and availability of innovative therapies, and addressing shortages and diversity in the cancer care workforce. Several overarching policy areas transcend across the delivery system, specifically the promotion of data-driven transparency, scalability, defining measures of quality and cost, and multidisciplinary buy in. Across the care delivery system, policies should facilitate transparent and meaningful data-sharing-including alternative data sources among providers, payers, and patients-to better coordinate, deliver, and measure high-quality cancer care. Under value-based payment models and delivery of innovative treatment, success will ultimately depend on quantifying measures of quality and cost. Moving the needle forward and generating a cultural shift in the delivery of value-based models of care, innovative therapies, and team-based coordination requires strong leadership among clinical, business, and government entities. Policies should facilitate standardized yet adaptable healthcare systems to promote the delivery of care that ensures quality and safety, patient-centered care, cost-effectiveness, evidencebased practice, team-based coordination, and access to innovation.

Submitted February 19, 2019; accepted for publication March 15, 2019.

Disclosures: Dr. Newcomer has disclosed that he serves on the board for Cellworks, and is a scientific advisor for Grail, Loxo Pharmaceuticals, Merrimack Pharmaceuticals, Mobil ODT, Reflexion Medical, RxFunction, Trakcel, Xenex. The remaining authors have disclosed that they have no financial interests, arrangements, affiliations, or commercial interests with the manufacturers of any products discussed in this article or their competitors. Ms. Winckworth-Prejsnar, Mr. McCanney, Ms. Schatz, Ms. Fitzgerald, and Dr. Carlson are employed by the National Comprehensive Cancer Network.

Correspondence: Katy Winckworth-Prejsnar, MPH, National Comprehensive Cancer Network, 3025 Chemical Road, Suite 100, Plymouth Meeting, PA 19462 Email: winckworthprejsnar@nccn.org 


\section{References}

1. Smedley W, Plantanias LC, Winckworth-Prejsnar K. Findings and recommendations of NCCN Policy Challenges and Opportunities to Address Changing Paradigms in Cancer Care Delivery Working Group. Presented at the NCCN Policy Summit: Policy Challenges and Opportunities to Address Changing Paradigms in Cancer Care Delivery; September 13, 2018; Washington DC.

2. Newcomer L. Good intentions and unintended consequences for cancer patients. Presented at the NCCN Policy Summit: Policy Challenges and Opportunities to Address Changing Paradigms in Cancer Care Delivery; September 13, 2018; Washington DC.

3. Policy solutions to delivering high-quality cancer care under value-based payment models. Panel discussion at the NCCN Policy Summit: Policy Challenges and Opportunities to Address Changing Paradigms in Cancer Care Delivery; September 13, 2018; Washington DC.

4. Miller HD; Center for Healthcare Quality \& Payment Reform. A better way to pay for cancer care. Available at: http://www.chqpr.org/ downloads/BetterPaymentforCancerCare.pdf. Accessed October 15, 2018

5. Department of Health and Human Services. Physician-Focused Payment Model Technical Advisory Committee value considerations for model development and testing fact cheet. Available at: https:// innovation.cms.gov/Files/fact-sheet/ptac-value-fs.pdf. Accessed December 5, 2018.

6. Guerrant L. Care coordination: the key to surviving in a value-based reimbursement world. Intermedix web site. Available at: https://www. intermedix.com/blog/care-coordination-the-key-to-surviving-in-avalue-based-reimbursement-world. Published July 3, 2018. Accessed December 18, 2018.

7. Institute of Medicine. Delivering high-quality cancer care: charting a new course for a system in crisis. Report brief. Available at: https://www.nap. edu/resource/18359/qualitycancercare_rb.pdf. Published September 2013. Accessed December 18, 2018.

8. Pearl R. Healthcare's dangerous fee-for service addition. Forbes. Available at: https://www.forbes.com/sites/robertpearl/2017/09/25/fee-forservice-addiction/\#4923c380c8ad. Published September 25, 2017. Accessed January 11, 2019

9. Oshima Lee E, Emanuel EJ. Shared decision making to improve care and reduce costs. N Engl J Med 2013;368:6-8.

10. Cancer Care Planning and Communications Act of 2018, HR 5160, 115th Cong, 2017-2018.

11. Patient Navigation Assistance Act of 2018, HR 6808, 115th Cong, 2017-2018.

12. Porter-Tacoronte AB. Optimizing the coordination of cancer care through best practices and policy. Presented at the NCCN Policy Summit: Policy Challenges and Opportunities to Address Changing Paradigms in Cancer Care Delivery; September 13, 2018; Washington, DC

13. Bennett K. GBR in Maryland-a new payment methodology for Maryland hospitals. Medicine Matters, Johns Hopkins Medicine. Available at: https:// medicine-matters.blogs.hopkinsmedicine.org/2015/11/gbr-in-maryland-a-new-payment-methodology-for-maryland-hospitals/ Published November 5, 2015. Accessed December 18, 2018.
14. DuVernay C. Interventions reduce unnecessary readmissions. BestPractice News, Office of Johns Hopkins Physicians. Johns Hopkins Medicine web site. Available at: https://www.hopkinsmedicine.org/office-of-johnshopkins-physicians/best-practice-news/interventions-reduceunnecessary-readmissions. Published February 9, 2018. Accessed December 18, 2018.

15. Verifying Accurate Leading-edge IVCT Development Act of 2018. Preliminary discussion draft. 115th Cong, 2nd Sess, 2018. Available at: https://degette.house.gov/sites/degette.house.gov/files/valid_act_ discussion_draft_12.6.18.pdf. Published December 6, 2018. Accessed December 18, 2018.

16. Gottlieb S, Shuren J, Silvis L. FDA proposes new steps to advance clinical testing to deliver new cures [FDA voices]. Silver Spring, MD; US Food and Drug Administration; December 6, 2018. Available at: https://www.fda. gov/NewsEvents/Newsroom/FDAVoices/ucm627742.htm. Accessed January 16, 2019.

17. Arndt RZ. FDA launches new office to rethink how drugs are reviewed. Modern Healthcare website. Available at: https://www. modernhealthcare.com/article/20190108/NEWS/190109918?utm_ source $=$ modernhealthcare\&utm campaign $=$ hits\&utm medium $=$ email\&utm_content $=$ 20190108-NEWS-190109918. Published January 8, 2019. Accessed January 10, 2019.

18. FDA Statement from FDA commissioner Scott Gottlieb, MD and Peter Marks, MD, PhD, director of the center for biologics evaluation and research on new policies to advance development of safe and effective cel and gene therapies [press release]. Silver Spring, MD; US Food and Drug Administration; January 15, 2019. Available at: https://www.fda.gov/ NewsEvents/Newsroom/PressAnnouncements/ucm629493.htm. Accessed January 15, 2019e.

19. California Future Health Workforce Commission. Meeting the demand for health: final report of the California Future Health Workforce Commission. Available at: https://futurehealthworkforce.org/wp-content/uploads/ 2019/03/MeetingDemandForHealthFinalReportCFHWC.pdf. Published February 2019. Accessed February 11, 2019.

20. Cavallo J. Increasing racial and ethnic diversity in the oncology workforce: a conversation with Karen M. Winkfield, MD, PhD. The ASCO Post, October 10, 2017. Available at: http://www.ascopost.com/issues/ october-10-2017/increasing-racial-and-ethnic-diversity-in-the-oncologyworkforce/. Accessed February 15, 2019.

21. American Society of Clinical Oncology. The state of cancer care in America, 2014: a report by the American Society of Clinical Oncology. J Oncol Pract 2014;10:119-142.

22. Sullivan T. Interstate medical licensure compact-expands to 17 states Policy \& Medicine website. Available at: https://www.policymed.com/ 2016/06/interstate-medical-licensure-compact-expands-to-17-states. html. Published May 5, 2019. Accessed January 1, 2019

23. MedPAC. Moving beyond the Merit-based Incentive Payment System; chapter 15. Report to the Congress: Medicare Payment Policy; March 2018:445-468. Available at: http://www.medpac.gov/docs/defaultsource/reports/mar18_medpac_ch15_sec.pdf?sfvrsn=0. Accessed December 21, 2018. 
Supplemental online content for:

\section{Policy Challenges and Opportunities to Address Changing Paradigms in Cancer Care Delivery}

Katy Winckworth-Prejsnar, MPH; James McCanney, JD; Alyssa A. Schatz, MSW; Warren Smedley, MSHA, MSHOS; Leonidas C. Platanias, MD, PhD; Cecil M. Benitez, PhD; Lee N. Newcomer, MD, MHA;

C. Lyn Fitzgerald, MJ; and Robert W. Carlson, MD

J Natl Compr Canc Netw 2019;17(5):424-431

eAppendix 1: NCCN Working Group Members: Policy Challenges and Opportunities to Address Changing Paradigms in Cancer Care Delivery 


\section{eAppendix 1. NCCN Working Group Members: Policy Challenges and Opportunities to Address Changing Paradigms in Cancer Care Delivery}

Leonidas C. Platanias, MD, PhD, Co-Chair, Robert H. Lurie Comprehensive Cancer Center of Northwestern University Warren Smedley, MSHA, MSHOS, Co-Chair, UAB Cancer Community Network, UAB

Cecil Benitez, PhD, Stanford Cancer Institute

Mara Bloom, JS, MS, MGH, Massachusetts General Hospital Cancer Center

Virginia Calega, MD, MBA, Independence Blue Cross

Robert W. Carlson, MD, National Comprehensive Cancer Network

Jennifer Carlson, BS, The Ohio State University

Lynette Cederquist, MD, UC San Diego

Devika Das, MD, University of Alabama Comprehensive Cancer Center

Christian G. Downs, MHA, JD, Association of Community Cancer Centers (ACCC)

Deborah Kamin, RN, PhD, Policy \& Advocacy, ASCO

Eric Konnick, MD, MS, University of Washington

Despina (Desi) C. Kotis, PharmD, Robert H. Lurie Comprehensive Cancer Center of Northwestern University

Paul Melmeyer, MPP, National Organization for Rare Disorders (NORD)

MiKaela Olsen, MS, APRN-CNS, AOCNS, Sidney Kimmel Comprehensive Cancer Center

John O'Shea, MD, The Heritage Foundation

Mary Pinder-Schenck, MD, Merck

Alyssa Schatz, MSW, National Comprehensive Cancer Network

Wendy H. Vogel, MSN, FNP, AOCNP, The American Society of Pediatric Hematology/Oncology (ASPHO) 